\title{
Performance Enhancement of SPD Technique in SAC-OCDMA Systems
}

\author{
Ruchita Bajpai ${ }^{1}$, Dr. Neelam Srivastava ${ }^{2}$ \\ ${ }^{1}$ (Electronics Department, Institute of Engineering and Technology, Lucknow, India) \\ 2 (Professor, Electronics Department, Institute of Engineering and Technology, Lucknow, India)
}

\begin{abstract}
This paper presents single photodiode as an effective technique for eradicating the effects of PIIN and MAI by cancelling interference effects in the optical domain in spectral amplitude coding optical codedivision multiple-access(SAC-OCDMA)systems. Single photodiode detection(SPD) technique proves to be a better candidate than MODIFIED-AND subtraction detection in terms of BER performance. Appreciable performance can be obtained with SPD technique by using optical hard limiter.
\end{abstract}

Keywords: MAI, OCDMA, PIIN, SAC-OCDMA ,SPD

\section{Introduction}

Multiple access techniques are needed to meet the demand for high-speed and large capacity communications in optical networks. This technique allows many users to share the fiber bandwidth. Each user is given a particular time slot in time-division multiplexing(TDM) and a particular frequency(wavelength) slot in wavelength -division multiplexing(WDM). Above techniques have been extensively explored and utilized in optical communication systems. Alternatively, optical-code division multiple access(OCDMA) is receiving increased attention due to its advantages of enhanced information security, simplified and decentralized network control, improved spectral efficiency and asynchronous access [1]. It has applications in metro and local area networks and fiber to home as well. OCDMA is an optical based multiple-access technique that assigns every user a specific code which ensures efficient and secure sharing of network resources among users[2]. At the same time, MAI(multiple access interference) is the primary degradation factor in conventional OCDMA systems. Among all the OCDMA techniques, SAC can suppress the first order MAI when codes with fixed in-phase cross correlation are used as address sequences, and receiver side consists of two photodiodes connected in balanced manner[3,4,5]. In SAC-OCDMA technique, a spectral encoding has been applied on the output of a broad-band source by decomposing it into spectral bins and the intensity of each bin is modulated such that the bin is either "on" or "off" depending on user code being applied. Each user has a unique code consisting of specific combination of spectral components, which should be orthogonal to all other user codes. The key to an effective SAC-OCDMA system is the choice of efficient codes for encoding the source bits. The task of choosing the most efficient code family is not simple because many properties differentiate them such as code length $(\mathrm{N})$, cross-correlation $\left(\lambda_{C}\right)$, code weight $(\mathrm{w})$, number of users( $(\mathrm{k})$.Incoherent broad-band sources such as light-emitting diode(LED) or an erbium-doped fiber source are the best choices for SAC-OCDMA system since they are inexpensive, have wide optical bandwidth and provide comparable performance as coherent sources[3]. Also, incoherent sources involve less complexity than coherent ones as they don't need synchronization schemes [6]. However, these sources are thermal in nature and thus suffer from phase-induced intensity noise(PIIN), which limits the throughput of SAC OCDMA systems. Of late, SPD technique is proposed to eliminate the effects of PIIN and MAI in SAC-OCDMA system through cancelling the interference signal in the optical domain [2] . The research on SPD technique is of great importance. In this study, modified doubleweight (MDW) codes are utilized as the signature sequences.

\section{Modified Double-Weight(Mdw) Code}

MDW is the modified version of double weight(DW) code. The MDW code weight can be any even number that is greater than 2 . MDW codes can also be represented by using $[\mathrm{K} \times \mathrm{N}]$ matrix with $\mathrm{K}$ as row and $\mathrm{N}$ as column. The basic MDW can be developed by using following steps:

Step 1:

The basic matrix for MDW codes also consists of a $[\mathrm{K} \times \mathrm{N}]$ matrixdepending on the value of code weight. The general form of matrix for a MDW code is shown below: 


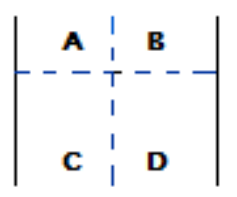

From the above matrix, the elements in each section are given by:
a. A consists of:
$\mathrm{A}=1 \times 3 \sum_{j=1}^{\frac{w}{2}-1} j$ matrix of zeroes

b. $\quad$ B consists of:

$\mathrm{B}=[1 \times 3 \mathrm{n}]$ matrix of basic matrix of $\left[X_{2}\right]$ for every 3 columns.

c. C consists of:

$\mathrm{C}$ is the basic code matrix for the next smaller weight, $\mathrm{w}=2(\mathrm{n}-1)$

d. D consists of:

$\mathrm{D}$ is a matrix $[\mathrm{n} \times \mathrm{n}]$ consisting of basic matrix of $\left[X_{3}\right]$ arranged as:

$\left[\begin{array}{lll}000 & 000 & {\left[X_{3}\right]} \\ 000 & {\left[X_{3}\right]} & 000 \\ {\left[X_{3}\right]} & 000 & 000\end{array}\right]$

A matrix $[\mathrm{n} \times \mathrm{n}]$ consisting of basic matrix of $\left[X_{3}\right]$ and

$\mathrm{n}=\frac{W}{2}, \mathrm{w}=2,4,6$

where $X_{1}, X_{2}, X_{3}$ are the $[1 \times 3]$ matrix and it consist of

$X_{2}=\left[\begin{array}{lll}0 & 1 & 1\end{array}\right]$

$X_{3}=\left[\begin{array}{lll}1 & 1 & 0\end{array}\right]$

$X_{1}=\left[\begin{array}{lll}0 & 0 & 0\end{array}\right]$

Step 2:

There are two basic components in basic matrix for MDW codes which are:-

Code length, $N_{B}=3 \sum_{j=1}^{\frac{W}{2}} j$

Number of user, $K_{B}=\frac{W}{2}+1$

(5) and (6) represent the basic matrix for MDW code. $N_{B}$ is the column(represent basic code length) and $K_{B}$ is the row(it represents basic number of users). The MDW consists of $\left[K_{B} \times N_{B}\right]$. The codewords could be pointed out in Table I. for multiuser, we can derive the codewords, code length, number of users and code weight from (1)-(6). The other option to obtain codewords is to use mapping technique. The benefit of mapping technique is that the code weight is fixed although the user is increased. The code length for mapping technique is-

Code length, $\mathrm{N}=3 \mathrm{~K}+\frac{8}{3}\left[\left(\sin \frac{K \pi}{3}\right)\right]^{2}$

For 6 users, we can use codewords in Table I to produce codewords as in Table II with basic matrix of [6×18] and fix $w=4$ using mapping technique[7,8,9].

TABLE I. MDW code for 3 users

\begin{tabular}{|l|l|}
\hline User 1 & 000011011 \\
\hline User 2 & 011000110 \\
\hline User 3 & 110110000 \\
\hline
\end{tabular}

TABLE II. MDW code for 6 users employing mapping technique

\begin{tabular}{|l|l|}
\hline User 1 & 000000000000011011 \\
\hline User 2 & 000000000011000110 \\
\hline User 3 & 000000000110110000 \\
\hline User 4 & 000011011000000000 \\
\hline User 5 & 011000110000000000 \\
\hline User 6 & 110110000000000000 \\
\hline
\end{tabular}




\subsection{Modified-AND Subtraction Detection}

\section{Detection Schemes}

The modified-AND subtraction detection allows longer transmission distance or higher data or a larger number of users as compared to AND subtraction detection technique. It divides the spectrum of used code sequence which proves advantageous in terms of suppressing the impacts of PIIN and MAI in incoherent SACOCDMA systems . Although, the number of filters are the same in both detection techniques. The SACOCDMA receiver diagram is shown in Fig. 1. The received optical signal reaches the splitter and is divided into 2 parts: one to the upper decoding branches and the other reaches AND-decoder through an attenuator. The attenuator makes interference signal incident equal power to each photo-detector when the desired user is inactive. The decoders are placed in parallel configuration. This structure divides the weight of used code sequence and reduces the optical power level during the decoding process. The decoder has a spectral response which is matched with the active user, whereas AND decoder has overlapped bins from different interferers[7]. The photodiodes PD and S-PD are connected in electrical opposition to provide a differential signal between decoded signals. The equivalent passband resulting from filtering is chosen to be $75 \%$ of data rate $R_{B}$.

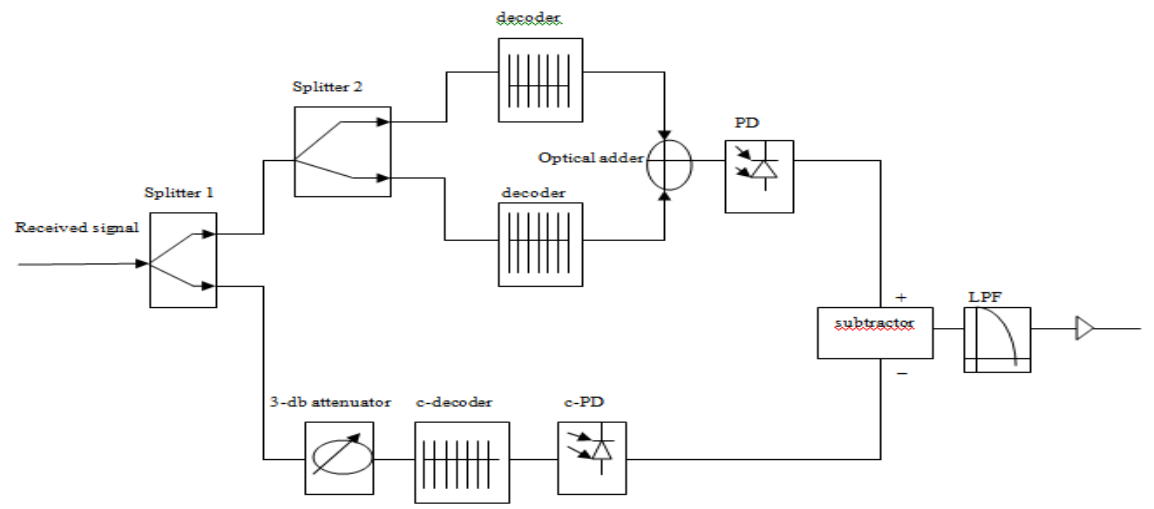

Fig. 1: Schematic diagram of Modified-AND subtraction detection technique

\subsection{Single Photodiode Detection(SPD)Technique}

The proposed SAC-OCDMA receiver diagram of this technique is shown in Fig.2. The received optical signal is decoded by the decoder, which has an identical spectral response to the intended encoder for the data to be received. The remainder of the signal from the decoder is then transmitted to the subtractive decoder(sdecoder) to cancel out signals with mismatched signatures i.e. interferers. The s-decoder contains only frequency bins from different interferers represented logically in Table III. The output from the s-decoder is either zero power unit for active user or cross-correlation power unit for interferers. The proposed technique can be performed using inexpensive ideal Fiber Bragg-gratings (FBGs)dispersion compensator to decode the received signal. After optical subtraction occurs, the output is either code weight power unit for active user or zero power unit for interferers. This shows that the interference signals are cancelled in the optical domain before conversion of signals to the electrical domain, as a result the SPD scheme alleviates both PIIN and MAI in the optical domain[10]. Theoretically, the two interference signals at the optical subtractor are assumed to be equal and cancel each other out. However, practically, the two interference signals differ slightly at the optical subtractor and as a result a small amount of optical power reaches photodiode. The use of single photodiode becomes possible because of cancellation of interference signals in the optical domain. Also, this reduces the amount of optical-to-electrical conversion and shot noise generated at the receiver side. This technique can also be implemented with any fixed in-phase cross-correlation SAC code with small amount of modification in the spectral distribution of s-Decoder, depending on the structure of SAC codes[11]. After the user signal is detected, the transmitted data are restored and filtered by the low-pass filter(LPF). The electrical LPFs are fourth-order Bessel filters with cut-off frequency of 0.75 of the data rate. 


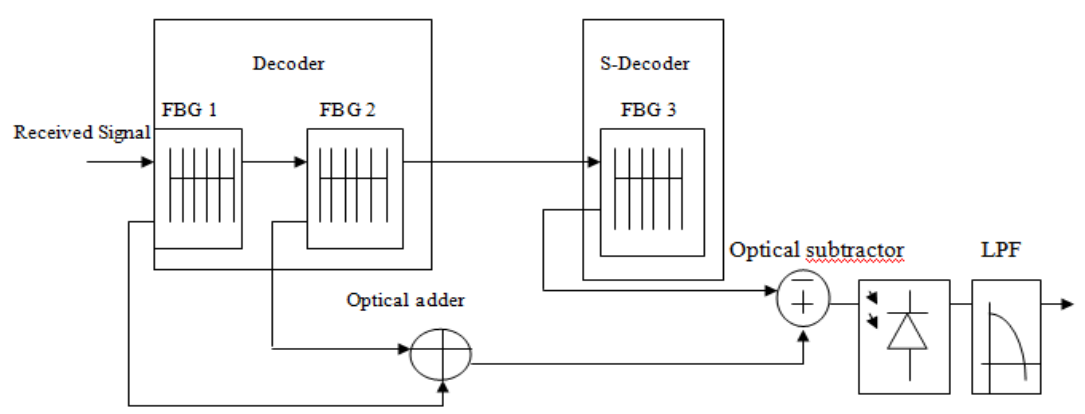

Fig. 2: SAC-OCDMA receiver based on SPD technique ;FBG: Fiber Bragg -grating; PD: photodiode

TABLE III. Logical Presentation Of Interference Cancellation For MDW code[11]

\begin{tabular}{|l|l|}
\hline & CODE WORDS \\
\hline MAIN USER(DEC) & 011000110 \\
\hline $1^{\text {st }}$ interfering user $\left(I_{1}\right)$ & 110110000 \\
\hline $2^{\text {nd }}$ interfering user $\left(I_{2}\right)$ & 000011011 \\
\hline$\left(\mathrm{DEC}{ }^{*} I_{1}\right)$ & 010000000 \\
\hline Sum $\left(I_{1}{ }^{*} \mathrm{DEC}\right)$ & 1 \\
\hline$(\mathrm{DEC}){ }^{\prime \prime \prime}$ & 100111001 \\
\hline$\left(I_{1}{ }^{*} I_{2}\right)$ & 000010000 \\
\hline s-DEC $=(\mathrm{DEC}))^{\prime \prime *}\left(I_{1}{ }^{*} I_{2}\right)$ & 000010000 \\
\hline$\left(I_{1}{ }^{*} \mathrm{DEC}\right)$ & 000010000 \\
\hline Sum $\left(I_{1}{ }^{*} \mathrm{~s}-\mathrm{DEC}\right)$ & 1 \\
\hline $\operatorname{Sum}\left(I_{1}{ }^{*} \mathrm{DEC}\right)-\operatorname{Sum}\left(I_{1}{ }^{*} \mathrm{~s}-\mathrm{DEC}\right)$ & $1-1=0$ \\
\hline
\end{tabular}

\section{Simulation Setup And Results}

The simulations of incoherent SAC-OCDMA have been performed in OptiSystem Version 9.0, which is very popular for conducting optical fiber simulations. To perform the tests one broad-band LED was taken and sliced into 20 wavelengths by WDM multiplexer for five active users. The bit rate for each user was 622 Mbps. The ITU-T G.652 standard single-mode optical fiber (SMF)was taken for $20 \mathrm{~km}$ optical transmission. Each chip has a spectral width of $0.8 \mathrm{~nm}$. The attenuation and dispersion were taken to be $0.25 \mathrm{db} / \mathrm{km}$ and $18 \mathrm{ps} / \mathrm{nm} \mathrm{km}$, respectively. For real simulation environment, nonlinear effects were activated and specified as close to the typical industrial values. The noises at the receiver section were taken random and totally uncorrelated. The dark current was set at $5 \mathrm{nA}$, and thermal noise coefficient was $1.8 \times 10^{-23} \mathrm{~W} / \mathrm{Hz}$ for each photodiode. The system performance is evaluated in terms of bit-error rate(BER) and calculation is done using Gaussian algorithm. All photodiodes taken are standard positive-intrinsic-negative(PIN) photodiodes with activated noises.

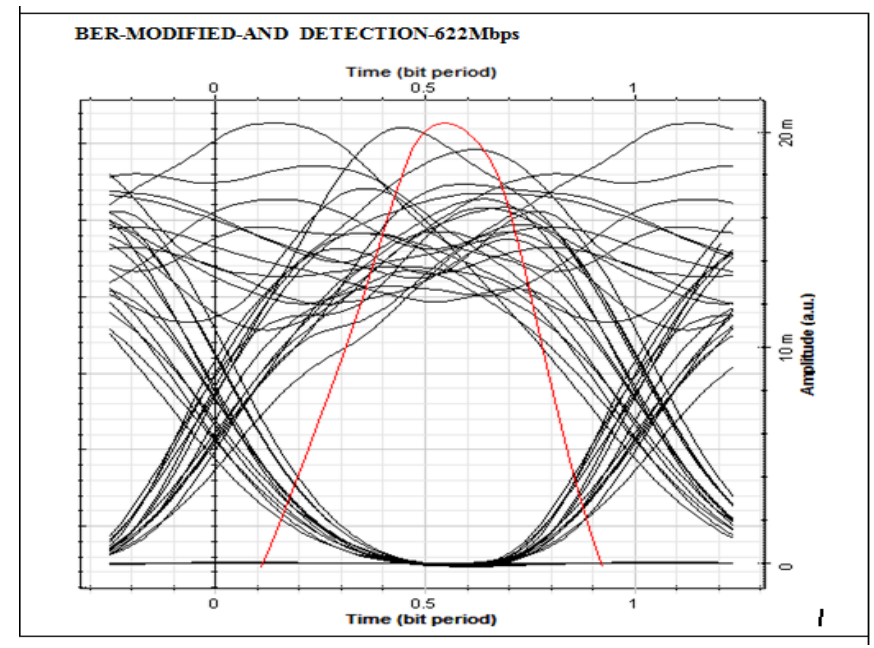

Fig. 3: EYE Diagram of MODIFIED-AND Subtraction Detection at $622 \mathrm{Mbps}$ 


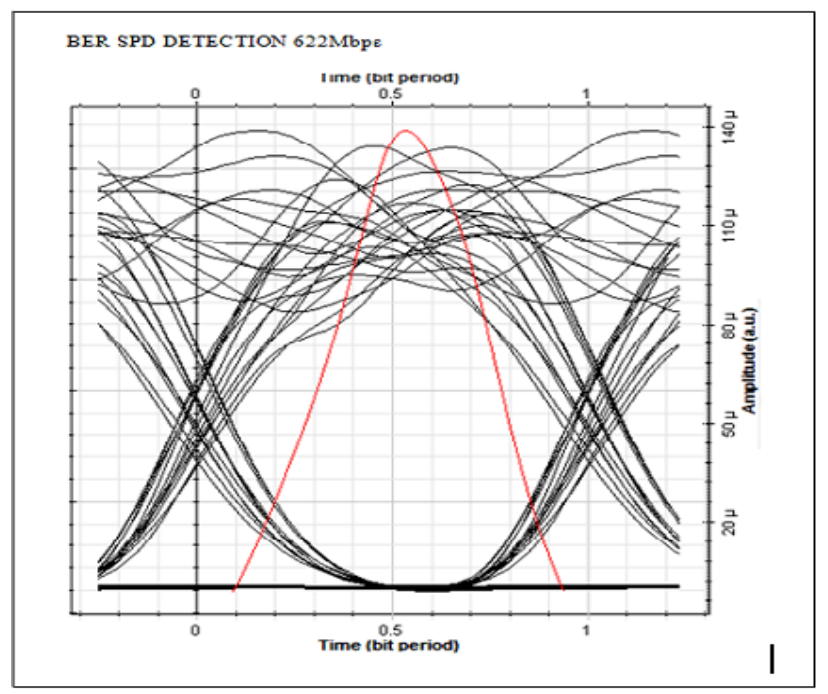

Fig.4: EYE diagram of SPD Technique at $622 \mathrm{Mbps}$

Fig .3 and Fig. 4 show the EYE diagrams of MODIFIED-AND subtraction and SPD detection techniques. On observing the EYE diagrams, it may be easily pointed out that SPD scheme achieves better BER performance than MODIFIED-AND subtraction detection scheme. The responsitivity of the photodetector is taken as $0.75 \mathrm{~A} / \mathrm{W}$ and quantum efficiency is 0.05 at a bandwidth of $30 \mathrm{~nm}$.

Practically, two interfering signals differ slightly at optical subtractor due to non-rectangular response of filters, which causes some form of distortion. Hence, to achieve appreciable performance , optical hard limiter(power threshold=-24 dbm; lower output level=-30 dbm ; upper output level= $-18 \mathrm{dbm}$ ) can be used. It is inserted between optical subtractor and PD so as to cancel the small amount of optical power reaching the PD [2]. Fig 5. Shows the eye diagram of SPD detection using optical hard limiter. It is observed that the BER performance is improved to a great extent with optical hard limiter.

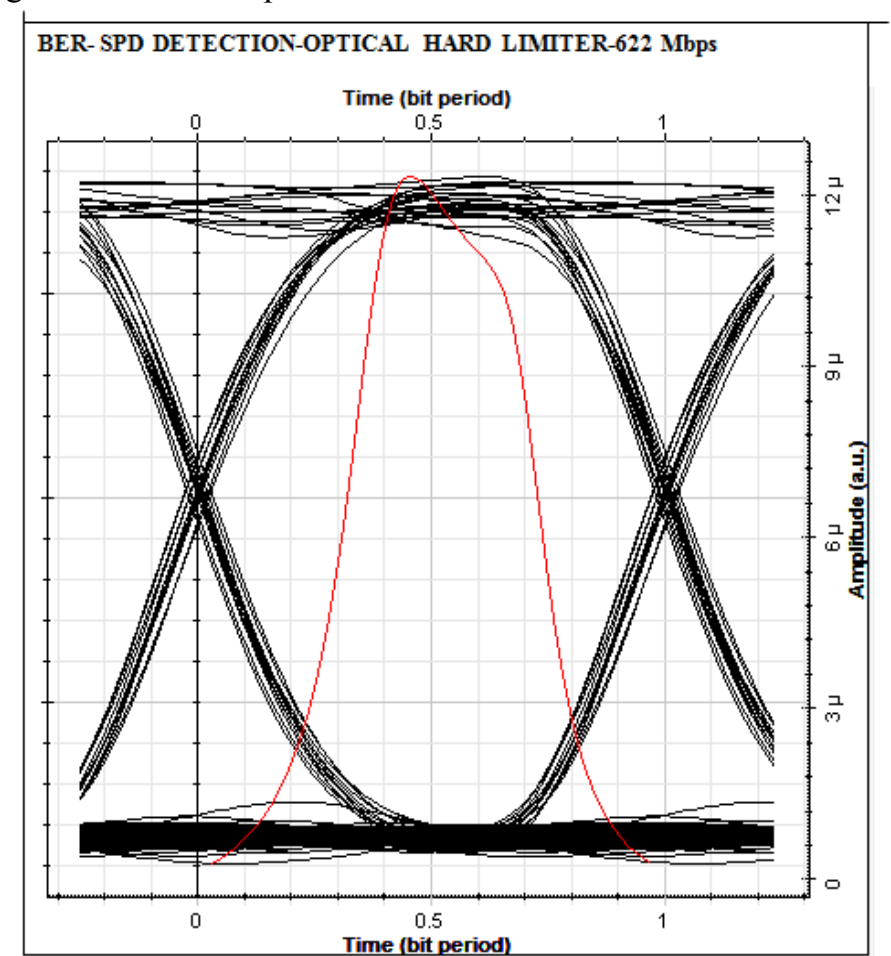

Fig. 5: EYE diagram of SPD detection using optical hard limiter at $622 \mathrm{Mbps}$

\section{Conclusion}

To mitigate the effects of PIIN and MAI, SPD detection technique is becoming a topic of research. The above results have validated the superiority of SPD technique over MODIFIED-AND subtraction detection 
technique in suppressing interference effects. As a result, BER performance of SPD technique is better than other detection schemes. Also, cost effectiveness is another advantage due to single photodiode instead of two in other detection techniques. Hence, SPD technique is an attractive scheme for future optical access networks.

\section{References}

[1]. Z.Jiang, D.S. Seo , S.D. Yang, D.E. Leaird, R.V. Roussev ,C. Langrock, M.M. Fejer, and A.M. Weiner, Four-User,2.5-Gb/s, spectrally coded OCDMA system demonstration using low-power nonlinear processing , Journal of Lightwave Technology,Vol.23,No.1,2005

[2]. Hamza M.R. Al-Khafaji, S.A. Aljunid, and Hilal A. Fadhil, Single photodiode detection for interference elimination in SACOCDMA systems , 978-1-4673-6075-3/13 IEEE,2013

[3]. Hamza M.R. Al-Khafaji, S.A. Aljunid, and Hilal A. Fadhil, Performance enhancement of SAC-OCDMA system using MODIFIED-AND subtraction detection, International Conference on Computer Applications and Industrial Electronics(ICCAIE),2011

[4]. M. Abtahi, S.Ayotte, J.Penon, L.A. Rusch, Balanced detection of correlated incoherent signals: a statistical analysis of intensity noise with experimental validation, J.Lightwave Technol., Vol.26,pp.1330-1338,2008

[5]. M. Noshad, K.Jamshidi , Code family for modified spectral-amplitude coding OCDMA systems and performance analysis, J.Opt.Commun.Netw, , Vol.2,pp.344-354, 2010

[6]. M.Z. Norazimah,S.A. Aljunid, Hamza M.R. Al-Khafaji, Hilal A.Fadhil and ,M.S. Anuar, Performance of different SAC-OCDMA detection schemes with NRZ and RZ data formats,2013 IEEE Symposium on Industrial Electronics \& Applications(ISIEA 2013),2013.

[7]. M.Z. Norazimah, Hamza M.R. Al-Khafaji, S.A. Aljunid and Hilal A. Fadhil, Performance comparison of different detection techniques in long-haul fiber SAC-OCDMA systems, $3^{\text {rd }}$ International Conference on Photonics 2012,2012

[8]. S.A Aljunid, M.Ismail, A.R.Ramli ,B.M. Ali, M.K.Abdullah, A new family of optical code sequences for spectral-amplitudecoding optical CDMA systems, IEEE Photon. Technol.Lett., Vol.16,pp.2383-2385,2004

[9]. S.A. Aljunid , Z.Zuraidah, A.A. SitiBarirah ,M.K. Abdullah, A new code for optical code division multiple access systems , Malaysian Journal of Computer Science,Vol.17,pp.30-39,2004

[10]. H.M.R. Al-Khafaji , S.A. Aljunid, A. Amphawan, H.A. Fadhil ,A.M. Safar, Reducing BER of spectral-amplitude coding optical code-division multiple access systems by single photodiode detection technique, J.Europ.opt.Soc.Rap.Public.8, 13022,2013

[11]. Sarah G. Abdulqader, S.A. Aljunid, Hamza M.R. Al-Khafaji ,Hilal A. Fadhil , Investigation of transmission performance for SACOCDMA system under long haul transmission distance based on single photodiode detection technique, 2013 IEEE $11^{\text {th }}$ Malaysia International Conference on Communications,Nov 2013

[12]. S.A. Aljunid, M.D.A. Samad, M. Othman, M.H. Hisham, A.H. Kasiman, and M.K. Abdullah, Development of modified doubleweight code and its implementation in multi-rate transmissions, IEEE,2005

[13]. Hamza M.R. Al-Khafaji, S.A. Aljunid, and Hilal A. Fadhil, Improved probability density function using Modified-AND detection technique for incoherent SAC-OCDMA systems, International Conference on Computer and Communication Engineering(ICCCE),2012

[14]. Hesham A. Bakarman, Sahbudin Shaari, Mahamod Ismail, Security performance of spectral amplitude coding OCDMA systems , International Conference on Electronic Design,2008 